\title{
Approximation algorithm for fixed points of nonlinear operators and solutions of mixed equilibrium problems and variational inclusion problems with applications
}

\author{
Uamporn Witthayarat ${ }^{a}$, Yeol Je Cho ${ }^{b, *}$, Poom Kumam ${ }^{a, *}$ \\ ${ }^{a}$ Department of Mathematics, Faculty of Science, King Mongkut's University of Technology Thonburi, KMUTT, Bangkok 10140, \\ Thailand. \\ ${ }^{b}$ Department of Mathematics Education and the RINS, Gyeongsang National University, Chinju 660-701, Korea.
}

Dedicated to George A Anastassiou on the occasion of his sixtieth birthday

Communicated by Professor R. Saadati

\begin{abstract}
The purpose of this paper is to introduce an iterative algorithm for finding a common element of the set of fixed point of nonexpansive mappings, set of a mixed equilibrium problem and the set of variational inclusions in a real Hilbert space. We prove that the sequence $x_{n}$ which is generated by the proposed iterative algorithm converges strongly to a common element of four sets above. Furthermore, we give an application to optimization and some numerical examples which support our main theorem in the last part. Our result extended and improve the existing result of Yao et al. [19] and references therein.
\end{abstract}

Keywords: Common fixed point; Equilibrium problem; Iterative algorithm; Nonexpansive mapping; Variational inequality.

\footnotetext{
${ }^{*}$ Corresponding author

Email addresses: u.witthayarat@hotmail.com (Uamporn Witthayarat), yjcho@gnu.ac.kr (Yeol Je Cho), poom.kum@kmutt.ac.th (Poom Kumam)
} 


\section{Introduction}

Throughout this paper, we assume that $H$ be a real Hilbert space with the inner product $\langle\cdot, \cdot\rangle$ and norm $\|\cdot\|$ and $C$ is a nonempty closed convex subset of $H$. Let $F: C \rightarrow H$ be a nonlinear mapping, $\varphi: C \rightarrow R$ be a function and $\Theta: C \times C \rightarrow R$ be a bifunction.

First, we consider the following mixed equilibrium problem: find $x^{*} \in C$ such that

$$
\Theta\left(x^{*}, y\right)+\varphi(y)-\varphi\left(x^{*}\right)+\left\langle F x^{*}, y-x^{*}\right\rangle \geq 0, \quad \forall y \in C .
$$

If $F=0$, then the mixed equilibrium problem (1.1) becomes the following mixed equilibrium problem, which was studied by Ceng and Yao [2]: Find $x^{*} \in C$ such that

$$
\Theta\left(x^{*}, y\right)+\varphi(y)-\varphi\left(x^{*}\right) \geq 0, \quad \forall y \in C .
$$

If $\varphi=0$, then the mixed equilibrium problem 1.1 becomes the following equilibrium problem, which was considered by Takahashi and Takahashi [16]: Find $x^{*} \in C$ such that

$$
\Theta\left(x^{*}, y\right)+\left\langle F x^{*}, y-x^{*}\right\rangle \geq 0, \quad \forall y \in C .
$$

If $\varphi=0$ and $F=0$, then the mixed equilibrium problem (1.1) becomes the following equilibrium problem: Find $x^{*} \in C$ such that

$$
\Theta\left(x^{*}, y\right) \geq 0, \quad \forall y \in C .
$$

If $\Theta(x, y)=0$ for all $x, y \in C$, then the mixed equilibrium problem (1.1) becomes the following variational inequality problem: Find $x^{*} \in C$ such that

$$
\varphi(y)-\varphi\left(x^{*}\right)+\left\langle F x^{*}, y-x^{*}\right\rangle \geq 0, \quad \forall y \in C .
$$

We denote the sets of solutions of the problems (1.1)-(1.5) by $E P(1)-E P(5)$, respectively. Equilibrium problem theory is one of most interesting and useful branch for the existence of solutions of many problems arising in economics, physics, operation research and other fields. The mixed equilibrium problems include fixed point problems, optimization problems, variational inequality problems, Nash equlibrium problems as the special cases. Also, many authors have introduced some kind of methods to solve these problems.

In 1997, Combettes and Hirstoaga [4] introduced an iterative method to find the best approximation to the initial data and also prove some strong convergence theorems by using the proposed method. Consequently, Takahashi and Takahashi [17] presented an another iterative scheme for finding a common element of the set of solutions of the equilibrium problem (1.2) and the set of fixed points of a nonexpansive mappings. Moreover, Yao, Liou and Yao [20, 21] also introduced new iterative schemes for finding a common element of the set of solutions of the equilibrium problem and the set of common fixed points of finitely (infinitely) nonexpansive mappings.

Recently, Ceng and Yao [2] introduced a new iterative scheme for finding a common element of the set of solution of the mixed equilibrium problem and the set of common fixed points of infinitely nonexpansive mappings. Furthermore, Peng and Yao [12] applied the CQ method to solve the mixed equilibrium problem and variational inequality problem and they also obtained the strong convergence results. Their result extended and improved the corresponding results in [3], [9], [16] and [21].

Recall that the mapping $f: C \rightarrow C$ is called a $\rho$-contraction if there exists a constant $\rho \in[0,1)$ such that

$$
\|f(x)-f(y)\| \leq \rho\|x-y\|, \quad \forall x, y \in C .
$$

A mapping $T: C \rightarrow C$ is said to be nonexpansive if

$$
\|T x-T y\| \leq\|x-y\|, \quad \forall x, y \in C .
$$


A mapping $B: C \rightarrow C$ is said to be $\beta$-inverse strongly monotone if there exists a constant $\beta>0$ such that

$$
\langle B x-B y, x-y\rangle \geq \beta\|B x-B y\|^{2}, \quad \forall x, y \in C .
$$

A mapping $G$ is said to be strongly positive on $H$ if there exists a constant $\mu>0$ such that

$$
\langle A x, x\rangle \geq \mu\|x\|^{2}, \quad \forall x \in H .
$$

Let $A: H \rightarrow H$ be a single-valued nonlinear mapping and $R: H \rightarrow 2^{H}$ be a set-valued mapping. The variational inclusion problem is as follows: Find $x \in H$ such that

$$
\theta \in B(x)+R(x)
$$

where $\theta$ is the zero vector in $H$. We denote the set of solution of this problem by $I(A, R)$.

Remark 1.1. (1) If $R=\partial \phi: H \rightarrow 2^{H}$ in (1.7), where $\phi: H \rightarrow R$ is proper convex lower semi-continuous and $\partial \phi$ is the sub-differential of $\phi$, then the variational inclusion (1.7) is equivalent to the following problem : Find $x \in H$ such that

$$
\langle B x, v-x\rangle+\phi(y)-\phi(x) \geq 0, \quad \forall v, y \in H,
$$

which is called the mixed quasi-variational inequality in Noor [10].

(2) Let $M=\partial \delta_{C}$ in (1.7), where $C$ is a nonempty closed convex subset of $H$ and $\delta_{C}: H \rightarrow[0, \infty)$ is the indicator function of $C$, i.e.,

$$
\delta_{C}(x)= \begin{cases}0, & x \in C \\ +\infty, & \text { otherwise. }\end{cases}
$$

Then the variational inclusion $(1.7)$ is equivalent to the following problem: Find $x \in H$ such that

$$
\langle B x, v-x\rangle \geq 0, \quad \forall v \in H,
$$

which is called Hartman-Stampacchia's variational inequality.

Remark 1.2. (1) If $H=R^{m}$, then the problem (1.7) becomes the generalized equation introduced by Robinson [13].

(2) If $B=0$, then the problem (1.7) becomes the inclusion problem introduced by Rockafellar [14].

The problem (1.7) is the most widely use for the study of optimal solutions in many related areas including mathematical programming, complementarity, variational inequalities, optimal control and many other fields. Many kinds of variational inclusions problems have been improved, extended and generalized in recent years by many authors.

In 2008, Zhang et al. [22] introduced an algorithm for finding a common solutions for quasi variational in clusion and fixed point problems, they also prove a strong convergence theorems for approximating this common elements under the suitable condition. Moreover, Kocourek et al. [7] presented a new iterative scheme for finding a common element of the set of solution to the problem (1.7) and the set of fixed points of nonexpansive, nonspreading and hybrid mappings in Hilbert spaces. Peng et al. [11] introduced another iterative algorithm by the viscosity approximate method for finding a common element of the set of solutions of a variational inclusion with a set-valued maximal monotone mapping and inverse strongly monotone mappings, the set of solutions of an equilibrium problem and the set of fixed points of a nonexpansive mapping.

Very recently, Yao et al. [19], proposed the following iterative algorithm:

$$
\left\{\begin{array}{l}
\Theta\left(u_{n}, y\right)+\varphi\left(y_{n}\right)-\varphi\left(u_{n}\right)+\frac{1}{r}\left\langle y-u_{n}, u_{n}-\left(x_{n}-r F x_{n}\right)\right\rangle \geq 0, \\
x_{n+1}=\alpha_{n}\left(u+\gamma f\left(x_{n}\right)\right)+\beta_{n} x_{n}+\left[\left(1-\beta_{n}\right) I-\alpha_{n}(I+\nu A)\right] W_{n} J_{R, \lambda}\left(z_{n}-r A z_{n}\right),
\end{array}\right.
$$


where $\left\{\alpha_{n}\right\},\left\{\beta_{n}\right\}$ are two real sequences in $[0,1]$. Furthermore, they also proved that the proposed above algorithm converges strongly to a common element of the set of solution of mixed equilibrium problem and the set of fixed point of nonexpansive mapping and the set of a variational inclusion in a real Hilbert space.

Motivated and inspired by the above works, in this paper, we propose an iterative algorithm which extend from Yao et al. [19] as follows:

$$
\left\{\begin{array}{l}
\Theta\left(u_{n}, y\right)+\varphi\left(y_{n}\right)-\varphi\left(u_{n}\right)+\frac{1}{r}\left\langle y-u_{n}, u_{n}-\left(x_{n}-r F x_{n}\right)\right\rangle \geq 0 \\
z_{n}=J_{R, s_{2}}\left(u_{n}-s_{2} B u_{n}\right) \\
y_{n}=J_{R, s_{1}}\left(z_{n}-s_{1} A z_{n}\right), \\
x_{n+1}=\alpha_{n}\left(u+\gamma f\left(x_{n}\right)\right)+\beta_{n} x_{n}+\left[\left(1-\beta_{n}\right) I-\alpha_{n}(I+\nu G)\right] W_{n} y_{n},
\end{array}\right.
$$

where $\left\{\alpha_{n}\right\}$ and $\left\{\beta_{n}\right\}$ are two real sequences in $[0,1]$. Furthermore, we prove the strong convergence theorem and give an illustrative example to support our main theorem.

\section{Preliminaries}

Let $H$ be a real Hilbert space and $C$ be a nonempty closed convex subset of $H$. It follows that

$$
\|x-y\|^{2}=\|x\|^{2}+\|y\|^{2}-2\langle x, y\rangle, \quad \forall x, y \in H .
$$

Recall that the nearest projection $P_{C}$ from $H$ to $C$ assigns to each $x \in H$, the unique point $P_{C} x \in C$ satisfying the property

$$
\left\|x-P_{C} x\right\|=\min _{y \in C}\|x-y\|,
$$

which is equivalent to the following inequality

$$
\left\langle x-P_{C} x, P_{C} x-y\right\rangle \geq 0, \quad \forall y \in C .
$$

A set-valued mapping $T: H \rightarrow 2^{H}$ is called monotone if, for all $x, y \in H, f \in T x$ and $g \in T y$ imply $\langle x-y, f-g\rangle \geq 0$. A monotone mapping $T: H \rightarrow 2^{H}$ is said to be maximal if its graph $G(T)$ is not properly contained in the graph of any other monotone mapping. It is well known that a monotone mapping $T$ is maximal if and only if, for all $(x, f) \in H \times H,\langle x-y, f-g\rangle \geq 0$ for all $(y, g) \in G(T)$ implies $f \in T x$.

Let a set valued mapping $R: H \rightarrow 2^{H}$ be a maximal monotone. We define a resolvent operator $J_{R, \lambda}$ generated by $R$ and $\lambda$ as follows:

$$
J_{R, \lambda}=(I+\lambda R)^{-1}(x), \quad \forall x \in H,
$$

where $\lambda$ is a positive number. It is easily to see that the resolvent operator $J_{R, \lambda}$ is single-valued, nonexpansive and 1-inverse strongly monotone and moreover, a solution of the problem (1.7) is a fixed point of the operator $J_{R, \lambda}(I-\lambda B)$ for all $\lambda>0$ (see, for example, [8]).

In this paper, we assume that a bifunction $\Theta: H \times H \rightarrow R$ and a convex function $\varphi: H \rightarrow R$ satisfy the following conditions:

(H1) $\Theta(x, x)=0$ for all $x \in H$;

(H2) $\Theta$ is monotone, i.e., $\Theta(x, y)+\Theta(y, x) \leq 0$ for all $x, y \in H$;

(H3) for any $y \in H, x \rightarrow \Theta(x, y)$ is weakly upper semi-continuous;

(H4) for any $x \in H, y \rightarrow \Theta(x, y)$ is convex and lower semi-continuous; 
(H5) for any $x \in H$ and $r>0$, there exists a bounded subset $D_{x} \subset H$ and $y_{x} \in H$ such that, for any $z \in H \backslash D_{x}$,

$$
\Theta\left(z, y_{x}\right)+\varphi\left(y_{x}\right)-\varphi(z)+\frac{1}{r}\left\langle y_{x}-z, z-x\right\rangle<0 .
$$

Next, we recall some lemmas which will be needed in the rest of this paper.

Lemma 2.1. [12] Let $H$ be a real Hilber space and $\Theta: H \times H \rightarrow R$ be a bifunction. Let $\varphi: H \rightarrow R$ be a proper lower semicontinuous and convex function. For any $r>0$ and $x \in H$, define a mapping $S_{r}: H \rightarrow H$ as follow: for all $x \in H$,

$$
S_{r}(x)=\left\{z \in H: \Theta(z, y)+\varphi(y)-\varphi(z)+\frac{1}{r}\langle y-z, z-x\rangle \geq 0, \forall y \in H\right\} .
$$

Assume that the condition (H1)-(H5) hold. Then we have the following:

(1) For each $x \in H, S_{r}(x) \neq 0$ and $S_{r}$ is single valued;

(2) $S_{r}$ is firmly nonexpansive, i.e., for any $x, y \in H$,

$$
\left\|S_{r} x-S_{r} y\right\|^{2} \leq\left\langle S_{r} x-S_{r} y, x-y\right\rangle
$$

(3) $\operatorname{Fix}\left(S_{r}\right)=\operatorname{EP}(1)$;

(4) EP(1) is closed and convex.

Lemma 2.2. [15] Let $\left\{x_{n}\right\}$ and $\left\{z_{n}\right\}$ be bounded sequences in a Banach space $E$ and $\left\{\beta_{n}\right\}$ be a sequence in $[0,1]$ satisfying the following condition:

$$
0<\liminf _{n \rightarrow \infty} \beta_{n} \leq \limsup _{n \rightarrow \infty} \beta_{n}<1 .
$$

Suppose that $x_{n+1}=\beta_{n} x_{n}+\left(1-\beta_{n}\right) z_{n}$ for all $n \geq 0$ and $\limsup _{n \rightarrow \infty}\left(\left\|z_{n+1}-z_{n}\right\|-\left\|x_{n+1}-x_{n}\right\|\right) \leq 0$. Then $\lim _{n \rightarrow \infty}\left\|z_{n}-x_{n}\right\|=0$.

Now, we define the mapping $W_{n}$ by

$$
\begin{aligned}
& U_{n, n+1}=I \\
& U_{n, n}=\lambda_{n} T_{n} U_{n, n+1}+\left(1-\lambda_{n}\right) I, \\
& U_{n, n-1}=\lambda_{n-1} T_{n-1} U_{n, n}+\left(1-\lambda_{n-1}\right) I, \\
& \quad \cdots \\
& U_{n, k}=\lambda_{k} T_{k} U_{n, k+1}+\left(1-\lambda_{k}\right) I, \\
& U_{n, k-1}=\lambda_{k-1} T_{k-1} U_{n, k}+\left(1-\lambda_{k-1}\right) I, \\
& \quad \cdots \\
& U_{n, 2}=\lambda_{2} T_{2} U_{n, 3}+\left(1-\lambda_{2}\right) I \\
& W_{n}=U_{n, 1}=\lambda_{1} T_{1} U_{n, 2}+\left(1-\lambda_{1}\right) I,
\end{aligned}
$$

where $\lambda_{1}, \lambda_{2}, \cdots$ are real numbers such that $0 \leq \lambda_{n} \leq 1$ for all $n \geq 1$ and $\left\{T_{i}\right\}_{n=1}^{\infty}$ is an infinite family of nonexpansive mappings $T_{n}: H \rightarrow H$.

Note that $W_{n}$ is usually called the $W$-mapping generated by $T_{n}, T_{n-1}, \cdots, T_{1}$ and $\lambda_{n}, \lambda_{n-1}, \cdots, \lambda_{1}$. It can be easily seen that $W_{n}$ is also nonexpansive mapping. 
Lemma 2.3. [15] Let $C$ be a nonempty closed convex subset of a real Hilbert space $H$. Let $\left\{T_{n}\right\}_{n=1}^{\infty}$ be an infinite family of nonexpanxive mappings $T_{n}: H \rightarrow H$ such that $\cap_{n=1}^{\infty} F i x\left(T_{n}\right) \neq 0$. Let $\lambda_{1}, \lambda_{2}, \cdots$ be a real number such that $0<\lambda_{n} \leq b<1$ for all $n \in N$. Then the following statements hold:

(1) For all $x \in H$ and $k \in N$, the limit $\lim _{n \rightarrow \infty} U_{n, k} x$ exists;

(2) $\operatorname{Fix}(W)=\cap_{n=1}^{\infty} \operatorname{Fix}\left(T_{n}\right)$ where $W x=\lim _{n \rightarrow \infty} W_{n} x=\lim _{n \rightarrow \infty} U_{n, 1} x$ for all $x \in C$;

(3) For any bounded sequence $\left\{x_{n}\right\}$ in $H$,

$$
\lim _{n \rightarrow \infty}\left\|W x_{n}-W_{n} x_{n}\right\|=0 .
$$

Lemma 2.4. [1] Let $R: H \rightarrow 2^{H}$ be a maximal monotone mapping and $B: H \rightarrow H$ be a Lipschitz and continuous monotone mapping. Then the mapping $R+B: H \rightarrow 2^{H}$ is maximal monotone.

Lemma 2.5. [5],[6] Let $\left\{a_{n}\right\}$ be a sequence of non-negative real numbers satisfying

$$
a_{n+1} \leq\left(1-\gamma_{n}\right) a_{n}+\delta_{n}, \quad n \geq 1,
$$

where $\left\{\gamma_{n}\right\}$ is a sequence in $(0,1)$ and $\left\{\delta_{n}\right\}$ satisfy the following conditions:

(i) $\sum_{n=1}^{\infty}=\infty$;

(ii) $\lim \sup _{n \rightarrow \infty} \frac{\delta_{n}}{\gamma_{n}} \leq 0$ or $\sum_{n=1}^{\infty}\left|\delta_{n}\right|<\infty$;

Then $\lim _{n \rightarrow \infty} a_{n}=0$.

Lemma 2.6. [5], [6] Let $C$ be a nonempty closed convex subset of a real Hilbert space $H$ and $g: C \rightarrow R \cup\{\infty\}$ be a proper lower-semicontinuous differentiable convex function. If $x^{*}$ is a solution to the minimization problem:

$$
g\left(x^{*}\right)=\inf _{x \in C} g(x)
$$

then

$$
\left\langle g^{\prime}(x), x-x^{*}\right\rangle \geq 0, \quad \forall x \in C .
$$

In particular, if $x^{*}$ solves the optimization problem:

$$
\min _{x \in C} \frac{\nu}{2}\langle A x, x\rangle+\frac{1}{2}\|x-u\|^{2}-h(x),
$$

then

$$
\left\langle u+(\gamma f-(I+\nu A)) x^{*}, x-x^{*}\right\rangle \leq 0 .
$$

\section{Main Results}

In this section, we prove some strong convergence theorems for finding a common element of the set of solution of a mixed equilibrium problem, the set of solution of variational inclusions and the set of fixed points of nonexpansive mappings. 
Theorem 3.1. Let $H$ a real Hilbert space and $\left\{T_{n}\right\}_{n=1}^{\infty}$ be an infinite family of nonexpansive mappings $T_{n}: H \rightarrow H$. Let $\varphi: H \rightarrow R$ be a lower semicontinuous and convex function and $\Theta: H \times H \rightarrow R$ be a bifunction satisfying the condition $(H 1)-(H 5)$. Let $G$ be a strongly positive bounded linear operator with coefficient $\mu>0$ and $R: H \rightarrow 2^{H}$ be a maximal monotone mapping. Let $A, B, F: H \rightarrow H$ be $\alpha, \beta, \eta$-inverse strongly monotone mappings, respectively. Let $f: H \rightarrow H$ be a $\rho$-contraction and $r>$ $0, s_{1}, s_{2}>0, \gamma>0$ be four constants such that $s_{1}<2 \alpha, s_{2}<2 \beta, r<2 \rho$ and $\gamma<\frac{(1+\nu) \mu}{\rho}$. Assume that $\Omega:=\left(\cap_{n=1}^{\infty} F i x\left(T_{n}\right)\right) \cap E P(1) \cap I(A, R) \cap I(B, R) \neq \emptyset$. Let $W_{n}$ be the mapping defined by (2.2). If $\left\{x_{n}\right\}$ is the sequence generated by $x_{1} \in H$ and

$$
\left\{\begin{array}{l}
\Theta\left(u_{n}, y\right)+\varphi\left(y_{n}\right)-\varphi\left(u_{n}\right)+\frac{1}{r}\left\langle y-u_{n}, u_{n}-\left(x_{n}-r F x_{n}\right)\right\rangle \geq 0, \\
z_{n}=J_{R, s_{2}}\left(u_{n}-s_{2} B u_{n}\right), \\
y_{n}=J_{R, s_{1}}\left(z_{n}-s_{1} A z_{n}\right), \\
x_{n+1}=\alpha_{n}\left(u+\gamma f\left(x_{n}\right)\right)+\beta_{n} x_{n}+\left[\left(1-\beta_{n}\right) I-\alpha_{n}(I+\nu G)\right] W_{n} y_{n},
\end{array}\right.
$$

where $\left\{\alpha_{n}\right\}$ and $\left\{\beta_{n}\right\}$ are two real sequences in $[0,1]$ satisfying the following conditions:

(C1) $\lim _{n \rightarrow \infty} \alpha_{n}=0$ and $\Sigma_{n=1}^{\infty} \alpha_{n}=\infty$;

(C2) $0<\liminf _{n \rightarrow \infty} \beta_{n}<\lim \sup _{n \rightarrow \infty} \beta_{n}<1$.

Then the sequence $\left\{x_{n}\right\}$ converges strongly to a point $x^{*} \in \Omega$, which solve the following optimization problem:

$$
\min _{x \in \Omega} \frac{\nu}{2}\langle G x, x\rangle+\frac{1}{2}\|x-u\|^{2}-h(x),
$$

where $h$ is a potential function for $\gamma f$.

Proof. We divide the proof of Theorem 3.1 into several steps.

Step 1. We show that $\left\{x_{n}\right\}$ is bounded. First, we note that $I-s_{1} A$ and $I-s_{2} B$ are nonexpansive for all $x, y \in C$ and

$$
\begin{aligned}
\left\|\left(I-s_{1} A\right) x-\left(I-s_{1} A\right) y\right\|^{2} & =\|x-y\|^{2}-2 s_{1}\langle x-y, A x-A y\rangle+s_{1}^{2}\|A x-A y\|^{2} \\
& \leq\|x-y\|^{2}-2 s_{1} \alpha\|A x-A y\|^{2}+s_{1}^{2}\|A x-A y\|^{2} \\
& =\|x-y\|^{2}-s_{1}\left(2 \alpha-s_{1}\right)\|A x-A y\|^{2} \\
& \leq\|x-y\|^{2} .
\end{aligned}
$$

Thus $I-s_{1} A$ is nonexpansive and so are $I-s_{2} B$ and $I-r F$. Let $p \in \Omega$. From (C1) and (C2), we assume that $\alpha_{n} \leq\left(1-\beta_{n}\right)(1+\nu\|G\|)^{-1}$ for all $n \geq 1$. Since $G$ is a linear bounded self-adjoint operator on $H$, we have

$$
\|G\|=\sup \{|\langle G x, x\rangle|: x \in H,\|x\|=1\}
$$

and

$$
\begin{aligned}
\left\langle\left(\left(1-\beta_{n}\right) I-\alpha_{n}(I+\nu G)\right) x, x\right\rangle & =\left\langle\left(1-\beta_{n}\right) x-\alpha_{n} x-\alpha_{n} \nu G x, x\right\rangle \\
& =1-\beta_{n}-\alpha_{n}-\alpha_{n} \nu\langle G x, x\rangle \\
& \geq 1-\beta_{n}-\alpha_{n}-\alpha_{n} \nu\|G\| \\
& \geq 0 .
\end{aligned}
$$

Thus we can see that $\left(1-\beta_{n}\right) I-\alpha_{n}(I+\nu G)$ is positive. Further, it follows that

$$
\begin{aligned}
\left\|\left(1-\beta_{n}\right) I-\alpha_{n}(I+\nu G)\right\| & =\sup \left\{\left\langle\left(\left(1-\beta_{n}\right) I-\alpha_{n}(I+\nu G)\right) x, x\right\rangle x \in H,\|x\|=1\right\} \\
& =\sup \left\{1-\beta_{n}-\alpha_{n}-\alpha_{n} \nu\langle G x, x\rangle, x \in H,\|x\|=1\right\} \\
& \leq 1-\beta_{n}-\alpha_{n}(1+\nu \mu) .
\end{aligned}
$$


From $z_{n}=J_{R, s}\left(u_{n}-s_{2} B u_{n}\right)$ for all $n \geq 0$, we can compute

$$
\begin{aligned}
\left\|z_{n}-p\right\| & =\left\|J_{R, s_{2}}\left(u_{n}-s_{2} B u_{n}\right)-J_{R, s_{2}}\left(p-s_{2} B p\right)\right\| \\
& \leq\left\|\left(u_{n}-p\right)-s_{2}\left(B u_{n}-B p\right)\right\| \\
& \leq\left\|u_{n}-p\right\|
\end{aligned}
$$

and

$$
\begin{aligned}
\left\|y_{n}-p\right\| & =\left\|J_{R, s_{1}}\left(z_{n}-s_{1} A z_{n}\right)-J_{R, s_{1}}\left(p-s_{1} A p\right)\right\| \\
& \leq\left\|\left(z_{n}-p\right)-s_{1}\left(A z_{n}-A p\right)\right\| \\
& \leq\left\|z_{n}-p\right\| \\
& \leq\left\|u_{n}-p\right\| .
\end{aligned}
$$

Letting $u_{n}=S_{r}\left(x_{n}-r F x_{n}\right)$ for all $n \geq 0$, by Lemma 2.1, we have

$$
\begin{aligned}
\left\|u_{n}-p\right\|^{2} & =\left\|S_{r}\left(x_{n}-r F x_{n}\right)-S_{r}(p-r F p)\right\|^{2} \\
& \leq\left\|x_{n}-p\right\|^{2}-2 r\left\langle x_{n}-p, F x_{n}-F p\right\rangle+r^{2}\left\|F x_{n}-F p\right\|^{2} \\
& \leq\left\|x_{n}-p\right\|^{2}-r(2 \rho-r)\left\|F x_{n}-F p\right\|^{2} \\
& \leq\left\|x_{n}-p\right\|^{2}
\end{aligned}
$$

and so $\left\|z_{n}-p\right\| \leq\left\|x_{n}-p\right\|$ and $\left\|y_{n}-p\right\| \leq\left\|x_{n}-p\right\|$. Thus we have

$$
\begin{aligned}
\left\|x_{n+1}-p\right\|= & \| \alpha_{n} u+\alpha_{n}\left(\gamma f\left(x_{n}\right)-(I+\nu G) p\right)+\beta_{n}\left(x_{n}-p\right) \\
& +\left[\left(1-\beta_{n}\right) I-\alpha_{n}(I+\nu G)\right]\left(W_{n} y_{n}-p\right) \| \\
\leq & \left(1-\beta_{n}-\alpha_{n}(1+\nu \mu)\right)\left\|y_{n}-p\right\|+\beta_{n}\left\|x_{n}-p\right\| \\
& +\alpha_{n}\|u\|+\alpha_{n}\|\gamma f(x-n)-(I+\nu G) p\| \\
\leq & \left(1-\alpha_{n}(1+\nu \mu)\right)\left\|x_{n}-p\right\|+\alpha_{n}\|u\|+\alpha_{n} \gamma \eta\left\|x_{n}-p\right\| \\
& +\alpha_{n}\|\gamma f(p)-(I+\nu G) p\| \\
= & {\left[1-\alpha_{n}(1+\nu \mu+\gamma \eta)\right]\left\|x_{n}-p\right\|+\alpha_{n}[\|\gamma f(p)-(I+\nu G) p\|+\|u\|] } \\
\leq & \max \left\{\left\|x_{0}-p\right\|, \frac{\|\gamma f(p)-(I+\nu G) p\|+\|u\|}{1+\nu \mu-\gamma p}\right\} .
\end{aligned}
$$

Hence $\left\{x_{n}\right\}$ is bounded and so are $\left\{u_{n}\right\},\left\{z_{n}\right\},\left\{y_{n}\right\},\left\{W_{n} y_{n}\right\},\left\{f\left(x_{n}\right)\right\}$ and $\left\{G W_{n} y_{n}\right\}$.

Step 2. We show that $\lim _{n \rightarrow \infty}\left\|x_{n+1}-x_{n}\right\|=0$. Define $x_{n+1}=\beta_{n}+\left(1-\beta_{n}\right) v_{n}$ for all $n \geq 0$, that is, $v_{n}=\frac{x_{n+1}-\beta_{n} x_{n}}{1-\beta_{n}}$ for all $n \geq 0$. It follows that

$$
\begin{aligned}
v_{n+1}-v_{n}= & \frac{x_{n+2}-\beta_{n+1} x_{n+1}}{1-\beta_{n+1}}-\frac{x_{n+1}-\beta_{n} x_{n}}{1-\beta_{n}} \\
= & \frac{\alpha_{n+1}}{1-\beta_{n+1}}\left[u+\gamma f\left(x_{n+1}\right)\right]-\frac{\alpha_{n}}{1-\beta_{n}}\left[u+\gamma f\left(x_{n}\right)\right]+W_{n+1} y_{n+1}-W_{n} y_{n} \\
& -\frac{\alpha_{n+1}}{1-\beta_{n+1}}(I+\nu G) W_{n+1} y_{n+1}+\frac{\alpha_{n}}{1-\beta_{n}}(I+\nu G) W_{n} y_{n} \\
= & \frac{\alpha_{n+1}}{1-\beta_{n+1}}\left[u+\gamma f\left(x_{n+1}\right)-(I+\nu G) W_{n+1} y_{n+1}\right] \\
& +\frac{\alpha_{n}}{1-\beta_{n}}\left[u+\gamma f\left(x_{n}\right)+(I+\nu G) W_{n} y_{n}\right] \\
& +W_{n+1} y_{n+1}-W_{n+1} y_{n}+W_{n+1} y_{n}-W_{n} y_{n}
\end{aligned}
$$


and so

$$
\begin{aligned}
\left\|v_{n+1}-v_{n}\right\|-\left\|x_{n+1}-x_{n}\right\| \leq & \frac{\alpha_{n+1}}{1-\beta_{n+1}}\left[\|u\|+\left\|\gamma f\left(x_{n+1}\right)\right\|+\left\|(I+\nu G) W_{n+1} y_{n+1}\right\|\right] \\
& +\frac{\alpha_{n}}{1-\beta_{n}}\left[\|u\|+\left\|\gamma f\left(x_{n}\right)\right\|+\left\|(I+\nu G) W_{n} y_{n}\right\|\right] \\
& +\left\|y_{n+1}-y_{n}\right\|+\left\|W_{n+1} y_{n}-W_{n} y_{n}\right\|-\left\|x_{n+1}-x_{n}\right\| .
\end{aligned}
$$

Since $T_{i}$ and $U_{n, i}$ are nonexpansive, it follows that

$$
\begin{aligned}
\left\|W_{n+1} y_{n}-W_{n} y_{n}\right\|= & \left\|\lambda_{1} T_{q} U_{n+1,2} y_{n}-\lambda_{1} T_{1} U_{n, 2} y_{n}\right\| \\
\leq & \lambda_{1}\left\|U_{n+1,2} y_{n}-U_{n, 2} y_{n}\right\| \\
= & \lambda_{1}\left\|\lambda_{2} T_{2} U_{n+1,3} y_{n}-\lambda_{2} T_{2} U_{n, 3} y_{n}\right\| \\
\leq & \lambda_{1} \lambda_{2}\left\|U_{n+1,3} y_{n}-U_{n, 3} y_{n}\right\| \\
& \vdots \\
\leq & \lambda_{1} \lambda_{2} \ldots \lambda_{n}\left\|U_{n+1, n+1} y_{n}-U_{n, n+1} y_{n}\right\| \\
\leq & M \prod_{i=1}^{n} \lambda_{i},
\end{aligned}
$$

where $M>0$ is a constant such that $\sup \left\{\left\|U_{n+1, n+1} y_{n}-U_{n, n+1} y_{n}\right\|: n \geq 0\right\} \leq M$.

Observe that

$$
\begin{aligned}
\left\|y_{n+1}-y_{n}\right\| & =\left\|J_{R, s_{1}}\left(z_{n+1}-s_{1} A z_{n+1}\right)-J_{R, s_{1}}\left(z_{n}-s_{1} A z_{n}\right)\right\| \\
& \leq\left\|\left(I-s_{1} A\right) z_{n+1}-\left(I-s_{1} A\right) z_{n}\right\| \\
& \leq\left\|z_{n+1}-z_{n}\right\| \\
& \leq\left\|J_{R, s_{2}}\left(u_{n+1}-s_{2} B u_{n+1}\right)-J_{R, s_{2}}\left(u_{n}-s_{2} B u_{n}\right)\right\| \\
& \leq\left\|u_{n+1}-u_{n}\right\| \\
& \leq\left\|S_{r}\left(x_{n+1}-r F x_{n+1}\right)-S_{r}\left(x_{n}-r F x_{n}\right)\right\| \\
& \leq\left\|x_{n+1}-x_{n}\right\| .
\end{aligned}
$$

Therefore, we have

$$
\begin{aligned}
\left\|v_{n+1}-v_{n}\right\|-\left\|x_{n+1}-x_{n}\right\| \leq & \frac{\alpha_{n+1}}{1-\beta_{n+1}}\left[\|u\|+\left\|\gamma f\left(x_{n+1}\right)\right\|+\left\|(I+\nu G) W_{n+1} y_{n+1}\right\|\right] \\
& +\frac{\alpha_{n}}{1-\beta_{n}}\left[\|u\|+\left\|\gamma f\left(x_{n}\right)\right\|+\left\|(I+\nu G) W_{n} y_{n}\right\|\right]+M \prod_{i=1}^{n} \lambda_{i},
\end{aligned}
$$

which implies that

$$
\limsup _{n \rightarrow \infty}\left(\left\|v_{n+1}-v_{n}\right\|-\left\|x_{n+1}-x_{n}\right\|\right) \leq 0
$$

Hence, by Lemma 2.2, it follows that $\lim _{n \rightarrow \infty}\left\|v_{n}-x_{n}\right\|=0$ and

$$
\begin{aligned}
\lim _{n \rightarrow \infty}\left\|v_{n}-x_{n}\right\| & =\lim _{n \rightarrow \infty}\left\|\frac{x_{n+1}-\beta_{n} x_{n}}{1-\beta_{n}}-x_{n}\right\| \\
& =\lim _{n \rightarrow \infty} \frac{\left\|x_{n+1}-x_{n}\right\|}{1-\beta_{n}}
\end{aligned}
$$

and so

$$
\lim _{n \rightarrow \infty}\left\|x_{n+1}-x_{n}\right\|=0
$$

Step 3. We show that

$$
\lim _{n \rightarrow \infty}\left\|F x_{n}-F p\right\|=0, \lim _{n \rightarrow \infty}\left\|B u_{n}-B p\right\|=0, \lim _{n \rightarrow \infty}\left\|A z_{n}-A p\right\|=0 .
$$


From (3.1), it follows that

$$
x_{n+1}=\alpha_{n}\left(u+\gamma f\left(x_{n}\right)\right)+\beta_{n} x_{n}+\left[\left(1-\beta_{n}\right) I-\alpha_{n}(I+\nu G)\right] W_{n} y_{n},
$$

which can be rewritten as

$$
x_{n+1}=\alpha_{n}\left(u+\gamma f\left(x_{n}\right)-(I+\nu G) W_{n} y_{n}\right)+\beta_{n}\left(x_{n}-W_{n} y_{n}\right)+W_{n} y_{n} .
$$

Observe that

$$
\begin{aligned}
\left\|x_{n}-W_{n} y_{n}\right\| & \leq\left\|x_{n}-x_{n+1}\right\|+\left\|x_{n+1}-W_{n} y_{n}\right\| \\
& \leq\left\|x_{n}-x_{n+1}\right\|+\alpha_{n}\left\|u+\gamma g\left(x_{n}\right)-(I+\nu G) W_{n} y_{n}\right\|+\beta_{n}\left\|x_{n}-W_{n} y_{n}\right\|
\end{aligned}
$$

and so

$$
\left\|x_{n}-W_{n} y_{n}\right\| \leq \frac{1}{1-\beta_{n}}\left\|x_{n}-x_{n+1}\right\|+\frac{\alpha_{n}}{1-\beta_{n}}\left\|u+\gamma f\left(x_{n}\right)-(I+\nu G) W_{n} y_{n}\right\| .
$$

Thus, from $\alpha_{n} \rightarrow 0$ and $\lim _{n \rightarrow \infty}\left\|x_{n+1}-x_{n}\right\|=0$, it follows that

$$
\lim _{n \rightarrow \infty}\left\|x_{n}-W_{n} y_{n}\right\|=0 .
$$

Since $I-r A, I-s B$ and $I-r F$ are nonexpansive, it follows from (3.3) that

$$
\begin{aligned}
\left\|y_{n}-p\right\|^{2} \leq & \left\|J_{R, s_{1}}\left(z_{n}-s_{1} A z_{n}\right)-J_{R, s_{1}}\left(p-s_{1} A p\right)\right\|^{2} \\
\leq & \left\|z_{n}-p\right\|^{2}+s_{1}\left(s_{1}-2 \alpha\right)\left\|A z_{n}-A p\right\|^{2} \\
\leq & \left\|J_{R, s_{2}}\left(u_{n}-s_{2} B u_{n}\right)-J_{R, s_{2}}\left(p-s_{2} B p\right)\right\|^{2}+s_{1}\left(s_{1}-2 \alpha\right)\left\|A z_{n}-A p\right\|^{2} \\
\leq & \left\|u_{n}-p\right\|^{2}+s_{2}\left(s_{2}-2 \beta\right)\left\|B u_{n}-B p\right\|^{2}+s_{1}\left(s_{1}-2 \alpha\right)\left\|A z_{n}-A p\right\|^{2} \\
\leq & \left\|x_{n}-p\right\|^{2}+r(r-2 \rho)\left\|F x_{n}-F p\right\|^{2}+s_{2}\left(s_{2}-2 \beta\right)\left\|B u_{n}-B p\right\|^{2} \\
& +s_{1}\left(s_{1}-2 \alpha\right)\left\|A z_{n}-A p\right\|^{2} .
\end{aligned}
$$

Thus we obtain

$$
\begin{aligned}
\left\|x_{n+1}-p\right\|^{2}= & \left\|\alpha_{n}\left(u+\gamma f\left(x_{n}\right)-(I+\nu G) p\right)+\beta_{n}\left(x_{n}-W_{n} y_{n}\right)+\left(I-\alpha_{n}(I+\nu G)\right)\left(W_{n} y_{n}-p\right)\right\|^{2} \\
\leq & \left\|\left(I-\alpha_{n}(I+\nu G)\right)\left(W_{n} y_{n}-p\right)+\beta_{n}\left(x_{n}-W_{n} y_{n}\right)\right\|^{2} \\
& +2 \alpha_{n}\left\langle u+\gamma f\left(x_{n}\right)-(I+\nu G) p, x_{n+1}-p\right\rangle \\
\leq & {\left[\left\|I-\alpha_{n}(I+\nu G)\right\|\left\|y_{n}-p\right\|+\beta_{n}\left\|x_{n}-W_{n} y_{n}\right\|\right]^{2} } \\
& \left.+2 \alpha_{n} \| u+\gamma f\left(x_{n}\right)-(I+\nu G) p\right)\|\| x_{n+1}-p \| \\
= & (1-\alpha-n(1+\nu \mu))^{2}\left\|y_{n}-p\right\|^{2}+\beta_{n}^{2}\left\|x_{n}-W_{n} y_{n}\right\|^{2} \\
& +2\left(1-\alpha_{n}(1+\nu \mu)\right) \beta_{n}\left\|y_{n}-p\right\|\left\|x_{n}-W_{n} y_{n}\right\| \\
& +2 \alpha_{n}\left\|u+\gamma f\left(x_{n}\right)-(I+\nu G) p\right\|\left\|x_{n+1}-p\right\| .
\end{aligned}
$$

Since $\left(1-\alpha_{n}(1+\nu \mu)\right)^{2}<1$, it follows that

$$
\begin{aligned}
\left\|x_{n+1}-p\right\|^{2} \leq & \left\|y_{n}-p\right\|^{2}+\beta_{n}^{2}\left\|x_{n}-W_{n} y_{n}\right\|^{2}+2\left(1-\alpha_{n}(1+\nu \mu)\right) \beta_{n}\left\|y_{n}-p\right\|\left\|x_{n}-W_{n} y_{n}\right\| \\
& +2 \alpha_{n}\left\|u+\gamma f\left(x_{n}\right)-(I+\nu G) p\right\|\left\|x_{n+1}-p\right\| \\
\leq & \left\|x_{n}-p\right\|^{2}+r(r-2 \rho)\left\|F x_{n}-F p\right\|^{2}+s_{2}\left(s_{2}-2 \beta\right)\left\|B u_{n}-B p\right\|^{2} \\
& +s_{1}\left(s_{1}-2 \alpha\right)\left\|A z_{n}-A p\right\|^{2} \beta_{n}^{2}\left\|x_{n}-W_{n} y_{n}\right\|^{2} \\
& +2\left(1-\alpha_{n}(1+\nu \mu)\right) \beta_{n}\left\|y_{n}-p\right\|\left\|x_{n}-W_{n} y_{n}\right\| \\
& +2 \alpha_{n}\left\|u+\gamma f\left(x_{n}\right)-(I+\nu G) p\right\|\left\|x_{n+1}-p\right\|,
\end{aligned}
$$


and so

$$
\begin{aligned}
r(2 \rho-r)\left\|F x_{n}-F p\right\|^{2}+ & s_{2}\left(2 \beta-s_{2}\right)\left\|B u_{n}-B p\right\|^{2}+s_{1}\left(2 \alpha-s_{1}\right)\left\|A z_{n}-A p\right\|^{2} \\
\leq & \left(\left\|x_{n}-p\right\|+\left\|x_{n+1}-p\right\|\right)\left(\left\|x_{n+1}-x_{n}\right\|\right)+\beta_{n}\left\|x_{n}-W_{n} y_{n}\right\|^{2} \\
& +2\left(1-\alpha_{n}(1+\nu \mu)\right) \beta_{n}\left\|y_{n}-p\right\|\left\|x_{n}-W_{n} y_{n}\right\| \\
& +2 \alpha_{n}\left\|u+\gamma f\left(x_{n}\right)-(I+\nu G) p\right\|\left\|x_{n+1}-p\right\| .
\end{aligned}
$$

Therefore, from (3.4), 3.5) and (3.7) it follows that

$$
\lim _{n \rightarrow \infty}\left\|F x_{n}-F p\right\|=0, \lim _{n \rightarrow \infty}\left\|B u_{n}-B p\right\|=0, \lim _{n \rightarrow \infty}\left\|A z_{n}-A p\right\|=0 .
$$

Step 4. We show that $\left\|x_{n}-W x_{n}\right\| \rightarrow 0$. Since $S_{r}$ is firmly nonexpansive, we have

$$
\begin{aligned}
\left\|u_{n}-p\right\|^{2}= & \left\|S_{r}\left(x_{n}-r F x_{n}\right)-S_{r}(p-r F p)\right\|^{2} \\
\leq & \left\langle x_{n}-r F x_{n}-(p-r F p), u_{n}-p\right\rangle \\
= & \frac{1}{2}\left(\left\|x_{n}-p\right\|^{2}+\left\|u_{n}-p\right\|^{2}-\left\|x_{n}-u_{n}-r\left(F x_{n}-F p\right)\right\|^{2}\right) \\
= & \frac{1}{2}\left(\left\|x_{n}-p\right\|^{2}+\left\|u_{n}-p\right\|^{2}-\left\|x_{n}-u_{n}\right\|^{2}+2 r\left\langle F x_{n}-F p, x_{n}-u_{n}\right\rangle\right. \\
& \left.-r^{2}\left\|F x_{n}-F p\right\|^{2}\right),
\end{aligned}
$$

which implies that

$$
\begin{aligned}
\left\|u_{n}-p\right\|^{2} & \leq\left\|x_{n}-p\right\|^{2}-\left\|x_{n}-u_{n}\right\|^{2}+2 r\left\langle F x_{n}-F p, x_{n}-u_{n}\right\rangle-r^{2}\left\|F x_{n}-F p\right\|^{2} \\
& =\left\|x_{n}-p\right\|^{2}-\left\|x_{n}-u_{n}\right\|^{2}+2 r\left\|F x_{n}-F p\right\|\left\|x_{n}-u_{n}\right\|-r^{2}\left\|F x_{n}-F p\right\|^{2} \\
& \leq\left\|x_{n}-p\right\|^{2}-\left\|x_{n}-u_{n}\right\|^{2}+2 r\left\|F x_{n}-F p\right\|\left\|x_{n}-u_{n}\right\| .
\end{aligned}
$$

Since $J_{R, s}$ is 1-inverse strongly monotone, we have

$$
\begin{aligned}
\left\|z_{n}-p\right\|^{2}= & \left\|J_{R, s_{2}}\left(u_{n}-s_{2} B u_{n}\right)-J_{R, s_{2}}\left(p-s_{2} B p\right)\right\|^{2} \\
\leq & \left.\left\langle\left(u-s_{2} B u_{n}\right)-\left(p-s_{2} B p\right), z_{n}-p\right)\right\rangle \\
= & \frac{1}{2}\left(\left\|\left(u_{n}-s_{2} B u_{n}\right)-\left(p-s_{2} B p\right)\right\|^{2}+\left\|z_{n}-p\right\|^{2}\right. \\
& \left.-\left\|\left(u_{n}-s_{2} B u_{n}\right)-\left(p-s_{2} B p\right)-\left(z_{n}-p\right)\right\|^{2}\right) \\
\leq & \frac{1}{2}\left(\left\|u_{n}-p\right\|^{2}+\left\|z_{n}-p\right\|^{2}-\left\|u_{n}-z_{n}-s_{2}\left(B u_{n}-B p\right)\right\|^{2}\right) \\
= & \frac{1}{2}\left(\left\|u_{n}-p\right\|^{2}+\left\|z_{n}-p\right\|^{2}-\left\|u_{n}-z_{n}\right\|^{2}+2 s_{2}\left\langle B u_{n}-B p, u_{n}-z_{n}\right\rangle\right. \\
& \left.-s_{2}^{2}\left\|B u_{n}-B p\right\|^{2}\right),
\end{aligned}
$$

which implies that

$$
\left\|z_{n}-p\right\|^{2} \leq\left\|u_{n}-p\right\|^{2}-\left\|u-n-z_{n}\right\|^{2}+2 s_{2}\left\|B u_{n}-B p\right\|\left\|u_{n}-z_{n}\right\| .
$$

Now, we observe that

$$
\begin{aligned}
\left\|y_{n}-p\right\|^{2}= & \left\|J_{R, s_{1}}\left(z_{n}-s_{1} A z_{n}\right)-J_{R, s_{1}}\left(p-s_{1} A p\right)\right\|^{2} \\
\leq & \left\langle z_{n}-s_{1} A z_{n}-\left(p-s_{1} A p\right), y_{n}-p\right\rangle \\
= & \frac{1}{2}\left(\left\|z_{n}-p\right\|^{2}+\left\|y_{n}-p\right\|^{2}-\left\|z_{n}-y_{n}-s_{1}\left(A z_{n}-A p\right)\right\|^{2}\right) \\
= & \frac{1}{2}\left(\left\|z_{n}-p\right\|^{2}+\left\|y_{n}-p\right\|^{2}-\left\|z_{n}-y_{n}\right\|^{2}+2 s_{1}\left\langle A z_{n}-A p, z_{n}-y_{n}\right\rangle\right. \\
& \left.-s_{1}^{2}\left\|A z_{n}-A p\right\|^{2}\right),
\end{aligned}
$$


that is,

$$
\begin{aligned}
\left\|y_{n}-p\right\|^{2} \leq & \left\|z_{n}-p\right\|^{2}-\left\|z_{n}-y_{n}\right\|^{2}+2 s_{1}\left\|A z_{n}-A p\right\|\left\|z_{n}-y_{n}\right\| \\
\leq & \left\|u_{n}-p\right\|^{2}-\left\|u_{n}-z_{n}\right\|^{2}+2 s_{2}\left\|B u_{n}-B p\right\|\left\|u_{n}-z_{n}\right\|-\left\|z_{n}-y_{n}\right\|^{2} \\
& +2 s_{1}\left\|A z_{n}-A p\right\|\left\|z_{n}-y_{n}\right\| \\
\leq & \left\|x_{n}-p\right\|^{2}-\left\|x_{n}-u_{n}\right\|^{2}+2 r\left\|F x_{n}-F p\right\|\left\|x_{n}-u_{n}\right\|-\left\|u_{n}-z_{n}\right\|^{2} \\
& +2 s_{2}\left\|B u_{n}-B p\right\|\left\|u_{n}-z_{n}\right\|-\left\|z_{n}-y_{n}\right\|^{2}+2 s_{1}\left\|A z_{n}-A p\right\|\left\|z_{n}-y_{n}\right\| .
\end{aligned}
$$

Substituting 3.8 in (3.6), we have

$$
\begin{aligned}
\left\|x_{n+1}-p\right\|^{2} \leq & \left(1-\alpha_{n}(1+\nu \mu)\right)^{2}\left\{\left\|x_{n}-p\right\|^{2}-\left\|x_{n}-u_{n}\right\|^{2}-\left\|u_{n}-z_{n}\right\|^{2}-\left\|z_{n}-y_{n}\right\|^{2}\right. \\
& +2 r\left\|F x_{n}-F p\right\|\left\|x_{n}-u_{n}\right\|+2 s_{2}\left\|B u_{n}-B p\right\|\left\|u_{n}-z_{n}\right\| \\
& \left.+2 s_{1}\left\|A z_{n}-A p\right\|\left\|z_{n}-y_{n}\right\|\right\} \\
& \beta_{n}^{2}\left\|x_{n}-W_{n} y_{n}\right\|^{2}+2\left(1-\alpha_{n}(1+\nu \mu)\right) \beta_{n}\left\|y_{n}-p\right\|\left\|x_{n}-W_{n} y_{n}\right\| \\
& +2 \alpha_{n}\left\|u+\gamma f\left(x_{n}\right)-(I+\nu G) p\right\|\left\|x_{n+1}-p\right\| .
\end{aligned}
$$

It follows that

$$
\begin{aligned}
& \left(1-\alpha_{n}(1+\nu \mu)\right)^{2} \quad\left\{\left\|x_{n}-u_{n}\right\|^{2}+\left\|u_{n}-z_{n}\right\|^{2}+\left\|z_{n}-y_{n}\right\|^{2}\right\} \\
& \leq\left(\left\|x_{n}-p\right\|+\left\|x_{n+1}-p\right\|\right)\left\|x_{n+1}-x_{n}\right\|+2 r\left\|F x_{n}-F p\right\|\left\|x_{n}-u_{n}\right\| \\
& +2 s_{2}\left\|B u_{n}-B p\right\|\left\|u_{n}-z_{n}\right\|+2 s_{1}\left\|A z_{n}-A p\right\|\left\|z_{n}-y_{n}\right\| \\
& +2\left(1-\alpha_{n}(1+\nu \mu)\right) \beta_{n}\left\|y_{n}-p\right\|\left\|x_{n}-W_{n} y_{n}\right\| \\
& +2 \alpha_{n}\left\|u+\gamma f\left(x_{n}\right)-(I+\nu G) p\right\|\left\|x_{n+1}-p\right\| \text {. }
\end{aligned}
$$

Thus we have

$$
\lim _{n \rightarrow \infty}\left\|x_{n}-u_{n}\right\|=0, \lim _{n \rightarrow \infty}\left\|u_{n}-z_{n}\right\|=0, \text { and } \lim _{n \rightarrow \infty}\left\|z_{n}-y_{n}\right\|=0 .
$$

Note that

$$
\left\|W y_{n}-y_{n}\right\| \leq\left\|W y_{n}-W_{n} y_{n}\right\|+\left\|W_{n} y_{n}-y_{n}\right\|
$$

and so, from this and Lemma 2.3 , it follows that $\lim _{n \rightarrow \infty}\left\|W y_{n}-y_{n}\right\|=0$. Therefore, we have

$$
\lim _{n \rightarrow \infty}\left\|x_{n}-W x_{n}\right\|=0 .
$$

Step 5. We show that

$$
\limsup _{n \rightarrow \infty}\left\langle u+\gamma f\left(x^{*}\right)-(I+\nu G) x^{*}, x_{n}-x^{*}\right\rangle \leq 0,
$$

where $x^{*}$ is a solution of the optimization problem. First, we note that there exists a subsequence $\left\{x_{n_{i}}\right\}$ of $\left\{x_{n}\right\}$ such that

$$
\limsup _{n \rightarrow \infty}\left\langle u+(\gamma f-(I+\nu G)) x^{*}, x_{n}-x^{*}\right\rangle=\lim _{j \rightarrow \infty}\left\langle u+(\gamma f-(I+\nu G)) x^{*}, x_{n_{j}}-x^{*}\right\rangle .
$$

Since $\left\{x_{n_{j}}\right\}$ is bounded, there exists a subsequence $\left\{x_{n_{j_{i}}}\right\}$ of $\left\{x_{n_{j}}\right\}$ which converges weakly to $\omega$. Without loss of generality, we can assume that $\left\{x_{n_{j}}\right\}$ converges weakly to the point $\omega$. Since $\left\|W x_{n}-x_{n}\right\| \rightarrow 0$, it follows the demiclosed principle of nonexpansive mappings that $\omega \in \operatorname{Fix}(W)$.

Now, we show that $\omega \in E P(1)$. By $u_{n}=S_{r}\left(x_{n}-r F x_{n}\right)$, it follows that

$$
\Theta\left(u_{n}, y\right)+\varphi(y)-\varphi\left(u_{n}\right)+\frac{1}{r}\left\langle y-u_{n}, u_{n}-\left(x_{n}-r F x_{n}\right)\right\rangle \geq 0, \quad \forall y \in H .
$$


From (H2), we have

$$
\varphi(y)-\varphi\left(u_{n}\right)+\frac{1}{r}\left\langle y-u_{n}, u_{n}-\left(x_{n}-r F x_{n}\right)\right\rangle \geq \Theta\left(y, u_{n}\right), \quad \forall y \in H,
$$

and hence

$$
\varphi(y)-\varphi\left(u_{n_{i}}\right)+\frac{1}{r}\left\langle y-u_{n_{i}}, u_{n_{i}}-\left(x_{n_{i}}-r F x_{n_{i}}\right)\right\rangle \geq \Theta\left(y, u_{n_{i}}\right), \quad \forall y \in H .
$$

For any $t \in(0,1]$ and $y \in H$, let $y_{t}=t y+(1-t) \omega$. From 33.9$)$, we have

$$
\begin{aligned}
& \varphi\left(y_{t}\right)-\varphi\left(u_{n_{i}}\right)+\frac{1}{r}\left\langle y_{t}-u_{n_{i}}, u_{n_{i}}-\left(x_{n_{i}}-r F x_{n_{i}}\right)\right\rangle \geq \Theta\left(y_{t}, u_{n_{i}}\right), \\
& \left\langle y_{t}-u_{n_{i}}, \frac{u_{n_{i}}-x_{n_{i}}+r F x_{n_{i}}}{r}\right\rangle \geq \Theta\left(y_{t}, u_{n_{i}}\right)-\varphi\left(y_{t}\right)+\varphi\left(u_{n_{i}}\right), \\
& \left\langle y_{t}-u_{n_{i}}, \frac{u_{n_{i}}-x_{n_{i}}+r F x_{n_{i}}}{r}+F\left(y_{t}\right)-F\left(y_{t}\right)\right\rangle \geq \Theta\left(y_{t}, u_{n_{i}}\right)-\varphi\left(y_{t}\right)+\varphi\left(u_{n_{i}}\right) .
\end{aligned}
$$

It follows that

$$
\begin{aligned}
\left\langle y_{t}-u_{n_{i}}, F y_{t}\right\rangle \geq & \varphi\left(u_{n_{i}}\right)-\varphi\left(y_{t}\right)+\left\langle y_{t}-u_{n_{i}}, F y_{t}\right\rangle+\Theta\left(y_{t}-u_{n_{i}}\right) \\
& -\left\langle y_{t}-u_{n_{i}}, \frac{u_{n_{i}}-x-n_{i}+r F x_{n_{i}}}{r}\right\rangle \\
= & \left\langle y_{t}-u_{n_{i}}, F y_{t}-F u_{n_{i}}\right\rangle+\left\langle y_{t}-u_{n_{i}}, F u_{n_{i}}-F x_{n_{i}}\right\rangle-\left\langle y_{t}-u_{n_{i}}, \frac{u_{n_{i}}-x_{n_{i}}}{r}\right\rangle \\
& +\varphi\left(u_{n_{i}}\right)-\varphi\left(y_{t}\right)+\Theta\left(y_{t}, u_{n_{i}}\right) .
\end{aligned}
$$

Since $\left\|u_{n_{i}}-x_{n_{i}}\right\| \rightarrow 0$, we have $\left\|F u_{n_{i}}-F x_{n_{i}}\right\| \rightarrow 0$. Further, from the inverse strongly monotonicity of $F$, it follows that $\left\langle y_{t}-u_{n_{i}}, F y_{t}-F u_{n_{i}}\right\rangle \geq 0$ and so, from (H4), the weakly lower semi-continuity of $\varphi$, $\frac{u_{n_{i}-x_{n_{i}}}}{r} \rightarrow 0$ and $u_{n_{i}} \rightarrow \omega$ weakly, it follows that

$$
\left\langle y_{t}-\omega, F y_{t}\right\rangle \geq-\varphi\left(y_{t}\right)+\varphi(\omega)+\Theta\left(y_{t}, \omega\right) .
$$

Thus, from (H1), (H4) and (3.10), we have

$$
\begin{aligned}
0 & =\Theta\left(y_{t}, y_{t}\right)-\varphi\left(y_{t}\right)+\varphi\left(y_{t}\right) \\
& =\Theta\left(y_{t}, t y+(1-t) \omega\right)-\varphi\left(y_{t}\right)+\varphi(t y+(1-t) \omega) \\
& \leq t\left[\Theta\left(y_{t}, y\right)+\varphi(y)-\varphi\left(y_{t}\right)\right]+(1-t)\left[\Theta\left(y_{t}, \omega\right)+\varphi(\omega)-\varphi\left(y_{t}\right)\right] \\
& \leq t\left[\Theta\left(y_{t}, y\right)+\varphi(y)-\varphi\left(y_{t}\right)\right]+(1-t)\left\langle y_{t}-\omega, F y_{t}\right\rangle \\
& =t\left[\Theta\left(y_{t}, y\right)+\varphi(y)-\varphi\left(y_{t}\right)\right]+(1-t) t\left\langle y-\omega, F y_{t}\right\rangle .
\end{aligned}
$$

Letting $t \rightarrow 0$, we have, for any $y \in H$,

$$
0 \leq \Theta(\omega, y)+\varphi(y)-\varphi(\omega)+\langle y-\omega, F \omega\rangle,
$$

which implies that $\omega \in E P(1)$.

Next, we show that $\omega \in I(B, R)$. Since $A$ be an $\alpha$-inverse strongly monotone, $A$ is Lipschitz continuous monotone mapping. It follows from Lemma (2.4) that $R+A$ is maximal monotone. Let $(v, g) \in G(R+A)$, i.e., $g-A v \in R(v)$. Since $y_{n_{i}}=J_{R, s_{1}}\left(z_{n_{i}}-s_{1} A z_{n_{i}}\right)$, we have $z_{n_{i}}-s_{1} z_{n_{i}} \in\left(I+s_{1} R\right) y_{n_{i}}$, i.e., $\frac{1}{s_{1}}\left(z_{n_{i}}-y_{n_{i}}-s_{1} A z_{n_{i}}\right) \in$ $R\left(y_{n_{i}}\right)$. By maximal monotonicity of $R+A$, we have

$$
\left\langle v-y_{n_{i}}, g-B v-\frac{1}{s_{1}}\left(z_{n_{i}}-y_{n_{i}}-s_{1} A z_{n_{i}}\right)\right\rangle \geq 0
$$

and so

$$
\begin{aligned}
\left\langle v-y_{n_{i}}, g\right\rangle & \geq\left\langle v-y_{n_{i}}, A v+\frac{1}{s_{1}}\left(z_{n_{i}}-y_{n_{i}}-s_{1} A z_{n_{i}}\right)\right\rangle \\
& \geq\left\langle v-y_{n_{i}}, A y_{n_{i}}-A z_{n_{i}}\right\rangle+\left\langle v-y_{n_{i}}, \frac{1}{s_{1}}\left(z_{n_{i}}-y_{n_{i}}\right)\right\rangle .
\end{aligned}
$$


It follows from $\left\|z_{n}-y_{n}\right\| \rightarrow 0,\left\|A z_{n}-A y_{n}\right\| \rightarrow 0$ and $y_{n_{i}} \rightarrow \omega$ weakly that

$$
\lim _{n_{i} \rightarrow \infty}\left\langle v-y_{n_{i}}, g\right\rangle=\langle v-\omega, g\rangle \geq 0 .
$$

Therefore, $\omega \in I(A, R)$ and, by the same method as in above, we can also find that $\omega \in I(B, R)$. Further, we have $\omega \in \Omega$ and

$$
\begin{aligned}
\limsup _{n \rightarrow \infty}\left\langle u+(\gamma f-(I+\nu G)) x^{*}, x_{n}-x^{*}\right\rangle & =\lim _{j \rightarrow \infty}\left\langle u+(\gamma f-(I+\nu G)) x^{*}, x_{n_{j}}-x^{*}\right\rangle \\
& =\left\langle u+(\gamma f-(I+\nu G)) x^{*}, \omega-x^{*}\right\rangle \\
& \leq 0 .
\end{aligned}
$$

Step 6. We show that the sequence $\left\{x_{n}\right\}$ converges to the point $x^{*}$. Observe that

$$
\begin{aligned}
\left\|x_{n+1}-x^{*}\right\|^{2}= & \left\|\alpha_{n}\left(u+\gamma f\left(x_{n}\right)\right)+\beta_{n} x_{n}+\left[\left(1-\beta_{n}\right) I-\alpha_{n}(I+\nu G)\right] W_{n} y_{n}-x^{*}\right\|^{2} \\
= & \| \alpha_{n}\left(u+\gamma f\left(x_{n}\right)-(I+\nu G) x^{*}\right)+\beta_{n}\left(x_{n}-x^{*}\right) \\
& +\left(\left(1-\beta_{n}\right) I-\alpha_{n}(I+\nu G)\right)\left(W_{n} y_{n}-x^{*}\right) \|^{2} \\
= & \left\|\beta_{n}\left(x_{n}-x^{*}\right)+\left(\left(1-\beta_{n}\right) I-\alpha_{n}(I+\nu G)\right)\left(W_{n} y_{n}-x^{*}\right)\right\|^{2} \\
& +2 \alpha_{n}\left\langle u+\gamma f\left(x_{n}\right)-(I+\nu G) x^{*}, x_{n+1}-x^{*}\right\rangle \\
\leq \quad & {\left[\left\|\left(\left(1-\beta_{n}\right) I-\alpha_{n}(I+\nu G)\right)\left(W_{n} y_{n}-x^{*}\right)\right\|+\left\|\beta_{n}\left(x_{n}-x^{*}\right)\right\|\right]^{2} } \\
& +2 \alpha_{n} \gamma\left\langle f\left(x_{n}\right)-f\left(x^{*}\right), x_{n+1}-x^{*}\right\rangle \\
& +2 \alpha_{n}\left\langle u+\gamma f\left(x^{*}\right)-(I+\nu G) x^{*}, x_{n+1}-x^{*}\right\rangle \\
\leq \quad & {\left[\left\|\left(\left(1-\beta_{n}\right) I-\alpha_{n}(I+\nu \mu)\right)\right\| y_{n}-x^{*}\left\|+\beta_{n}\right\| x_{n}-x^{*} \|\right]^{2} } \\
& +2 \alpha_{n} \gamma \rho\left\|x_{n}-x^{*}\right\|\left\|x_{n+1}-x^{*}\right\| \\
& +2 \alpha_{n}\left\langle u+\gamma f\left(x^{*}\right)-(I+\nu G) x^{*}, x_{n+1}-x^{*}\right\rangle \\
\leq & \left(1-\alpha_{n}(1+\nu) \mu\right)^{2}\left\|x_{n}-x^{*}\right\|^{2}+\alpha_{n} \gamma \rho\left\{\left\|x_{n}-x^{*}\right\|^{2}+\left\|x_{n+1}-x^{*}\right\|^{2}\right\} \\
& +2 \alpha_{n}\left\langle u+\gamma f\left(x^{*}\right)-(I+\nu G) x^{*}, x_{n+1}-x^{*}\right\rangle
\end{aligned}
$$

and so

$$
\begin{aligned}
\left\|x_{n+1}-x^{*}\right\| \leq & \frac{\left(1-\alpha_{n}(1+\nu) \mu\right)^{2}}{1-\alpha_{n} \gamma \rho}\left\|x_{n}-x^{*}\right\|^{2}+\frac{\alpha_{n} \gamma \rho}{1-\alpha_{n} \gamma \rho}\left\|x_{n}-x^{*}\right\|^{2} \\
& +\frac{2 \alpha_{n}}{1-\alpha_{n} \gamma \rho}\left\langle u+\gamma f\left(x^{*}\right)-(I+\nu G) x^{*}, x_{n+1}-x^{*}\right\rangle \\
= & {\left[1-\frac{2 \alpha_{n}((1+\nu) \mu-\gamma \rho)}{1-\alpha_{n} \gamma \rho}\left\|x_{n}-x^{*}\right\|^{2}+\frac{\left(\alpha_{n}\left(1_{\nu}\right) \mu\right)^{2}}{1-\alpha_{n} \gamma \rho}\left\|x_{n}-x^{*}\right\|^{2}\right.} \\
& +\frac{2 \alpha_{n}}{1-\alpha_{n} \gamma \rho}\left\langle u+\gamma f\left(x^{*}\right)-(I+\nu G) x^{*}, x_{n+1}-x^{*}\right\rangle \\
= & {\left[1-\frac{2 \alpha_{n}((1+\nu) \mu-\gamma \rho)}{1-\alpha_{n} \gamma \rho}\left\|x_{n}-x^{*}\right\|^{2}+\frac{2 \alpha_{n}((1+\nu) \mu-\gamma \rho)}{1-\alpha_{n} \gamma \rho}\right.} \\
& \times\left\{\frac{\alpha_{n}\left(\left(1_{\nu}\right) \mu\right)^{2}}{1-\alpha_{n} \gamma \rho} M_{1}+\frac{1}{\left(1_{\nu}\right) \mu-\gamma \rho}\left\langle u+\gamma f\left(x^{*}\right)-(I+\nu G) x^{*}, x_{n+1}-x^{*}\right\rangle\right\} \\
= & \left(1-\delta_{n}\right)\left\|x_{n}-x^{*}\right\|^{2}+\delta_{n} \alpha_{n},
\end{aligned}
$$

where

$$
\begin{gathered}
M_{1}=\sup \left\{\left\|x_{n}-x^{*}\right\|^{2}: n \geq 1\right\}, \quad \delta_{n}=\frac{2 \alpha_{n}((1+\nu) \mu-\gamma \rho)}{1-\alpha_{n} \gamma \rho} \\
\sigma_{n}=\frac{\alpha_{n}\left(\left(1_{\nu}\right) \mu\right)^{2}}{1-\alpha_{n} \gamma \rho} M_{1}+\frac{1}{\left(1_{\nu}\right) \mu-\gamma \rho}\left\langle u+\gamma f\left(x^{*}\right)-(I+\nu G) x^{*}, x_{n+1}-x^{*}\right\rangle .
\end{gathered}
$$


It can be easily seen that $\sum_{n=1}^{\infty} \delta_{n}=\infty$ and $\lim \sup _{n \rightarrow \infty} \sigma_{n} \leq 0$. Hence, by Lemma 2.5, we conclude that the sequence $\left\{x_{n}\right\}$ converges strongly to the point $x^{*} \in \Omega$. This completes the proof.

Using Theorem 3.1, we can obtain the following corollaries:

Corollary 3.2. For any $x_{0} \in H$, let $\left\{x_{n}\right\}$ be the sequence in $H$ generated by the following iterative algorithm: for any $y \in H$ and $n \geq 1$

$$
\left\{\begin{array}{l}
\Theta\left(u_{n}, y\right)+\varphi\left(y_{n}\right)-\varphi\left(u_{n}\right)+\frac{1}{r}\left\langle y-u_{n}, u_{n}-\left(x_{n}-r F x_{n}\right)\right\rangle \geq 0 \\
z_{n}=J_{R, s_{2}}\left(u_{n}-s_{2} B u_{n}\right), \\
y_{n}=J_{R, s_{1}}\left(z_{n}-s_{1} A z_{n}\right), \\
x_{n+1}=\alpha_{n}\left(u+\gamma f\left(x_{n}\right)\right)+\beta_{n} x_{n}+\left(1-\beta_{n}-\alpha_{n}\right) W_{n} y_{n},
\end{array}\right.
$$

where $\left\{\alpha_{n}\right\}$ and $\left\{\beta_{n}\right\}$ are two real sequences in $[0,1]$ satisfying the following conditions:

(C1) $\lim _{n \rightarrow \infty} \alpha_{n}=0$ and $\Sigma_{n=1}^{\infty} \alpha_{n}=\infty$;

(C2) $0<\liminf _{n \rightarrow \infty} \beta_{n}<\lim \sup _{n \rightarrow \infty} \beta_{n}<1$.

Suppose that $\Omega:=\left(\cap_{n=1}^{\infty} F i x\left(T_{n}\right)\right) \cap E P(1) \cap I(A, R) \cap I(B, R) \neq \emptyset$ and the mapping $W_{n}$ is defined by (2.2). Then the sequence $\left\{x_{n}\right\}$ converges strongly to a point $x^{*} \in \Omega$, which solves the following variational inequality:

$$
\left\langle u+\gamma f\left(x^{*}\right)-x^{*}, y-x\right\rangle \leq 0, \quad \forall y \in \Omega .
$$

Corollary 3.3. For any $x_{0} \in H$, let $\left\{x_{n}\right\}$ be the sequence in $H$ generated by the following iterative algorithm: for any $y \in H$ and $n \geq 1$

$$
\left\{\begin{array}{l}
\Theta\left(u_{n}, y\right)+\varphi\left(y_{n}\right)-\varphi\left(u_{n}\right)+\frac{1}{r}\left\langle y-u_{n}, u_{n}-x_{n}\right\rangle \geq 0 \\
z_{n}=J_{R, s_{2}}\left(u_{n}-s_{2} B u_{n}\right) \\
y_{n}=J_{R, s_{1}}\left(z_{n}-s_{1} A z_{n}\right) \\
x_{n+1}=\alpha_{n} \gamma f\left(x_{n}\right)+\beta_{n} x_{n}+\left[\left(1-\beta_{n}\right) I-\alpha_{n} G\right] W_{n} y_{n}
\end{array}\right.
$$

where $\left\{\alpha_{n}\right\}$ and $\left\{\beta_{n}\right\}$ are two real sequences in $[0,1]$ satisfying the following conditions:

(C1) $\lim _{n \rightarrow \infty} \alpha_{n}=0$ and $\Sigma_{n=1}^{\infty} \alpha_{n}=\infty$;

(C2) $0<\liminf _{n \rightarrow \infty} \beta_{n}<\lim \sup _{n \rightarrow \infty} \beta_{n}<1$.

Suppose that $\Omega:=\left(\cap_{n=1}^{\infty} F i x\left(T_{n}\right)\right) \cap E P(2) \cap I(A, R) \cap I(B, R) \neq \emptyset$ and the mapping $W_{n}$ is defined by (2.2). Then the sequence $\left\{x_{n}\right\}$ converges strongly to $x^{*} \in \Omega$, where $x^{*}=P_{\Omega}\left(\gamma f\left(x^{*}\right)+(I-G) x^{*}\right)$, which solves the following variational inequality:

$$
\langle\gamma f(x)-G x, y-x\rangle \leq 0, \quad \forall y \in \Omega
$$

Corollary 3.4. For any $x_{0} \in H$, let $\left\{x_{n}\right\}$ be the sequence in $H$ generated by the following iterative algorithm: for any $y \in H$ and $n \geq 1$

$$
\left\{\begin{array}{l}
\Theta\left(u_{n}, y\right)+\varphi\left(y_{n}\right)-\varphi\left(u_{n}\right)+\frac{1}{r}\left\langle y-u_{n}, u_{n}-\left(x_{n}-r F x_{n}\right)\right\rangle \geq 0 \\
z_{n}=J_{R, s_{2}}\left(u_{n}-s_{2} B u_{n}\right), \\
y_{n}=J_{R, s_{1}}\left(z_{n}-s_{1} A z_{n}\right), \\
x_{n+1}=\alpha_{n} \gamma f\left(x_{n}\right)+\beta_{n} x_{n}+\left[\left(1-\beta_{n}\right) I-\alpha_{n} G\right] y_{n},
\end{array}\right.
$$

where $\left\{\alpha_{n}\right\}$ and $\left\{\beta_{n}\right\}$ are two real sequences in $[0,1]$, which satisfy the following conditions: 
(C1) $\lim _{n \rightarrow \infty} \alpha_{n}=0$ and $\Sigma_{n=1}^{\infty} \alpha_{n}=\infty$;

(C2) $0<\liminf _{n \rightarrow \infty} \beta_{n}<\lim \sup _{n \rightarrow \infty} \beta_{n}<1$.

Suppose that $\Omega:=E P(1) \cap I(A, R) \cap I(B, R) \neq \emptyset$ and the mapping $W_{n}$ is defined by (2.2). Then the sequence $\left\{x_{n}\right\}$ converges strongly to $x^{*} \in \Omega$, where $x^{*}=P_{\Omega}\left(\gamma f\left(x^{*}\right)+(I-G) x^{*}\right)$, which solves the following variational inequality:

$$
\langle\gamma f(x)-G x, y-x\rangle \leq 0, \quad \forall y \in \Omega
$$

Corollary 3.5. For any $x_{0} \in H$, let $\left\{x_{n}\right\}$ be the sequence in $H$ generated by the following iterative algorithm: for any $y \in H$ and $n \geq 1$

$$
\left\{\begin{array}{l}
\Theta\left(u_{n}, y\right)+\frac{1}{r}\left\langle y-u_{n}, u_{n}-\left(x_{n}-r F x_{n}\right)\right\rangle \geq 0, \\
z_{n}=J_{R, s_{2}}\left(u_{n}-s_{2} B u_{n}\right), \\
y_{n}=J_{R, s_{1}}\left(z_{n}-s_{1} A z_{n}\right), \\
x_{n+1}=\alpha_{n} \gamma f\left(x_{n}\right)+\beta_{n} x_{n}+\left[\left(1-\beta_{n}\right) I-\alpha_{n} G\right] W_{n} y_{n},
\end{array}\right.
$$

where $\left\{\alpha_{n}\right\}$ and $\left\{\beta_{n}\right\}$ are two real sequences in $[0,1]$ satisfying the following conditions:

(C1) $\lim _{n \rightarrow \infty} \alpha_{n}=0$ and $\Sigma_{n=1}^{\infty} \alpha_{n}=\infty$;

(C2) $0<\liminf _{n \rightarrow \infty} \beta_{n}<\lim \sup _{n \rightarrow \infty} \beta_{n}<1$.

Suppose that $\Omega:=\left(\cap_{n=1}^{\infty} F i x\left(T_{n}\right)\right) \cap E P(3) \cap I(A, R) \cap I(B, R) \neq \emptyset$ and the mapping $W_{n}$ is defined by (2.2). Then the sequence $\left\{x_{n}\right\}$ converges strongly to $x^{*} \in \Omega$, where $x^{*}=P_{\Omega}\left(\gamma f\left(x^{*}\right)+(I-G) x^{*}\right)$, which solves the following variational inequality:

$$
\langle\gamma f(x)-G x, y-x\rangle \leq 0, \quad \forall y \in \Omega
$$

Corollary 3.6. For any $x_{0} \in H$, let $\left\{x_{n}\right\}$ be the sequence in $H$ generated by the following iterative algorithm: for any $y \in H$ and $n \geq 1$

$$
\left\{\begin{array}{l}
\Theta\left(u_{n}, y\right)+\frac{1}{r}\left\langle y-u_{n}, u_{n}-\left(x_{n}-r F x_{n}\right)\right\rangle \geq 0, \\
z_{n}=J_{R, s_{2}}\left(u_{n}-s_{2} B u_{n}\right), \\
y_{n}=J_{R, s_{1}}\left(z_{n}-s_{1} A z_{n}\right), \\
x_{n+1}=\alpha_{n} \gamma f\left(x_{n}\right)+\beta_{n} x_{n}+\left[\left(1-\beta_{n}\right) I-\alpha_{n} G\right] y_{n},
\end{array}\right.
$$

where $\left\{\alpha_{n}\right\}$ and $\left\{\beta_{n}\right\}$ are two real sequences in $[0,1]$ satisfying the following conditions:

(C1) $\lim _{n \rightarrow \infty} \alpha_{n}=0$ and $\Sigma_{n=1}^{\infty} \alpha_{n}=\infty$;

(C2) $0<\liminf _{n \rightarrow \infty} \beta_{n}<\lim \sup _{n \rightarrow \infty} \beta_{n}<1$.

Suppose that $\Omega:=E P(3) \cap I(A, R) \cap I(B, R) \neq \emptyset$ and the mapping $W_{n}$ is defined by 2.2. Then the sequence $\left\{x_{n}\right\}$ converges strongly to $x^{*} \in \Omega$, where $x^{*}=P_{\Omega}\left(\gamma f\left(x^{*}\right)+(I-A) x^{*}\right)$, which solves the following variational inequality:

$$
\langle\gamma f(x)-G x, y-x\rangle \leq 0, \quad \forall y \in \Omega .
$$


Corollary 3.7. For any $x_{0} \in H$, let $\left\{x_{n}\right\}$ be the sequence in $H$ generated by the following iterative algorithm: for any $y \in H$ and $n \geq 1$

$$
\left\{\begin{array}{l}
\Theta\left(u_{n}, y\right)+\frac{1}{r}\left\langle y-u_{n}, u_{n}-\left(x_{n}-r F x_{n}\right)\right\rangle \geq 0 \\
z_{n}=J_{R, s_{2}}\left(u_{n}-s_{2} B u_{n}\right) \\
y_{n}=J_{R, s_{1}}\left(z_{n}-s_{1} A z_{n}\right) \\
x_{n+1}=\alpha_{n} f\left(x_{n}\right)+\beta_{n} x_{n}+\left(1-\beta_{n}-\alpha_{n}\right) y_{n}
\end{array}\right.
$$

where $\left\{\alpha_{n}\right\}$ and $\left\{\beta_{n}\right\}$ are two real sequences in $[0,1]$ satisfying the following conditions:

(C1) $\lim _{n \rightarrow \infty} \alpha_{n}=0$ and $\Sigma_{n=1}^{\infty} \alpha_{n}=\infty$;

(C2) $0<\liminf _{n \rightarrow \infty} \beta_{n}<\lim \sup _{n \rightarrow \infty} \beta_{n}<1$.

Suppose that $\Omega:=E P(3) \cap I(A, R) \cap I(B, R) \neq \emptyset$ and the mapping $W_{n}$ is defined by 2.2). Then the sequence $\left\{x_{n}\right\}$ converges strongly to $x^{*} \in \Omega$, where $x *=P_{\Omega} f\left(x^{*}\right)$, which solves the following variational inequality:

$$
\langle f(x)-x, y-x\rangle \leq 0, \forall y \in \Omega .
$$

\section{Numerical Examples}

In this section, we give a real numerical example of the Main Theorem as follows:

Example 4.1. For simplicity, we assume $H=\mathbb{R}$ and $C=[-1,1]$. Let $\Theta(z, y)=-7 z^{2}+z y+6 y^{2}, F=I$ and $\phi(x)=x^{2}$. Find $z \in[-1,1]$ such that

$$
\Theta(z, y)+\phi(y)-\phi(z)+\frac{1}{r}\langle y-z, z-(x-r x)\rangle \geq 0, \quad \forall y \in[-1,1] .
$$

Solution. It can easily be seen that $\Theta, \phi$ and $F$ are satisfied the conditions in Theorem 3.1. For any $r>0$ and $x \in[-1,1]$, by Lemma 2.1, we can see that there exists $z \in[-1,1]$ such that, for any $y \in[-1,1]$,

$$
\begin{aligned}
& \Theta(z, y)+\phi(y)-\phi(z)+\frac{1}{r}\langle y-z, z-(x-r x)\rangle \geq 0, \\
& -7 z^{2}+6 y^{2}+z y+y^{2}-z^{2}+\frac{1}{r}\langle y-z, z-(x-r x)\rangle \geq 0, \\
& 7 r y^{2}+(r z+z-x+r x) y+\left(-8 r z^{2}-z^{2}+x z-r x z\right) \geq 0 .
\end{aligned}
$$

Let $H(y)=7 r y^{2}+(r z+z-x+r x) y+\left(-8 r z^{2}-z^{2}+x z-r x z\right)$. Then $H$ is a quadratic function of $y$ with coefficient $a=7 r, b=r z+z-x+r x$ and $c=-8 r z^{2}-z^{2}+x z-r x z$. Therefore, we can compute the discriminant $\Delta$ of $H$ as follows:

$$
\begin{aligned}
\Delta & =b^{2}-4 a c \\
& =[r z+z-x+r x]^{2}-4(7 r)\left(-8 r z^{2}-z^{2}+x z-r x z\right) \\
& =225 r^{2} z^{2}+30 r z^{2}-28 r x z+30 r^{2} x z+z^{2}-2 z x-2 r x^{2}+r^{2} x^{2}+x^{2} \\
& =\left(225 r^{2}+30 r+1\right) z^{2}+\left(30 r^{2}-28 r-2\right) x z+\left(r^{2}-2 r+1\right) x^{2} \\
& =(15 r+1)^{2} z^{2}+2(15 r+1)(r-1) x z+(r-1)^{2} x^{2} \\
& =[(15 r+1) z+(r-1) x]^{2} .
\end{aligned}
$$

We know that $H(y) \geq 0$ for all $y \in[-1,1]$ if it has at most one solution in $[-1,1]$. Thus $\Delta \leq 0$ and hence $z=\left(\frac{1-r}{15 r+1}\right) x$. Then we have $z=S_{r} x_{n}=\left(\frac{1-r}{15 r+1}\right) x_{n}$. Let $\left\{x_{n}\right\}$ be the sequence generated by 
$x_{1}=x \in[-1,1]$ and

$$
\left\{\begin{array}{l}
\Theta\left(u_{n}, y\right)+\varphi\left(y_{n}\right)-\varphi\left(u_{n}\right)+\frac{1}{r}\left\langle y-u_{n}, u_{n}-\left(x_{n}-r F x_{n}\right)\right\rangle \geq 0, \\
z_{n}=J_{R, s_{2}}\left(u_{n}-s_{2} B u_{n}\right), \\
y_{n}=J_{R, s_{1}}\left(z_{n}-s_{1} A z_{n}\right), \\
x_{n+1}=\alpha_{n}\left(u+\gamma f\left(x_{n}\right)\right)+\beta_{n} x_{n}+\left[\left(1-\beta_{n}\right) I-\alpha_{n}(I+\nu G)\right] W_{n} y_{n} .
\end{array}\right.
$$

Assume that $J_{R, s_{1}}, J_{R, s_{2}}, G=I, A x=\frac{x}{3}, B x=\frac{x}{2}$ and $W_{n}=\frac{x_{n}}{2}$.

Algorithm 1. Choose $r=0.5, \alpha_{n}=\frac{1}{10 n}, \beta_{n}=\frac{n}{2 n+1}, f\left(x_{n}\right)=\frac{1}{10} x_{n}$ and $s_{1}=s_{2}=\frac{1}{3}, \gamma=1, \nu=\frac{1}{2}, u=0$. Then the algorithm 4.1 becomes

$$
\left\{\begin{array}{l}
u_{n}=\frac{3}{17} x_{n} \\
z_{n}=\frac{1}{3} u_{n} \\
y_{n}=\frac{2}{9} z_{n} \\
x_{n+1}=\frac{x_{n}}{100 n}+\frac{n x_{n}}{2 n+1}+\left(\frac{n+1}{2 n+1}\right) \frac{y_{n}}{2}-\frac{y_{n}}{20 n}, \quad \forall n \geq 1
\end{array}\right.
$$

We can see that $x_{n} \rightarrow 0$ as $n \rightarrow \infty$, where 0 is the unique solution of the optimization problem:

$$
\min \frac{7}{10} x^{2}+C
$$

Algorithm 2. Choose $r=0.5, \alpha_{n}=\frac{1}{50 n}, \beta_{n}=\frac{n}{20 n+5}$. Then the algorithm 4.1 becomes

$$
\left\{\begin{array}{l}
u_{n}=\frac{3}{17} x_{n} \\
z_{n}=\frac{1}{3} u_{n} \\
y_{n}=\frac{2}{9} z_{n} \\
x_{n+1}=\frac{x_{n}}{500 n}+\frac{n x_{n}}{20 n+5}+\left(\frac{19 n+5}{20 n+5}\right) \frac{y_{n}}{2}-\frac{y_{n}}{20 n}, \quad \forall n \geq 1
\end{array}\right.
$$

\section{Numerical result.}

In this part we give the numerical results that support our main theorem as shown by calculating and plotting graphs using Matlab 7.11.0.

\section{Algorithm 1.}

\begin{tabular}{|c|c|}
\hline$n$ & $x_{n}$ \\
\hline 1 & 0.500000000000000 \\
2 & 0.173518518518519 \\
3 & 0.070898759380295 \\
4 & 0.030870860056218 \\
5 & 0.013904609765372 \\
6 & 0.006395839590864 \\
$\vdots$ & $\vdots$ \\
47 & 0.000000000000001 \\
48 & 0.000000000000001 \\
49 & 0.000000000000000 \\
\hline
\end{tabular}


Figure 1. This table shows the value of sequence $\left\{x_{n}\right\}$ on each iteration steps.

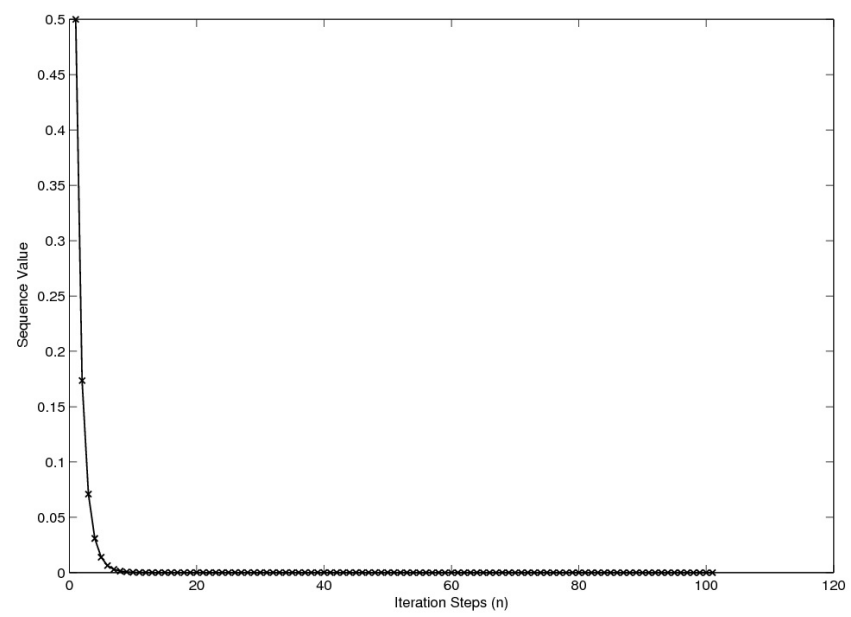

Figure 2. This figure shows the graph of the above table, we can see that $x_{n}$ converges to zero. Algorithm 2.

\begin{tabular}{|c|c|}
\hline$n$ & $x_{n}$ \\
\hline 1 & 0.500000000000000 \\
2 & 0.023810457516340 \\
3 & 0.001222979105852 \\
4 & 0.000064618469337 \\
5 & 0.000003465087782 \\
6 & 0.000000187506436 \\
$\vdots$ & $\vdots$ \\
11 & 0.000000000000093 \\
12 & 0.000000000000005 \\
13 & 0.000000000000000 \\
\hline
\end{tabular}

Figure 3. This table shows the value of sequence $\left\{x_{n}\right\}$ on each iteration steps.

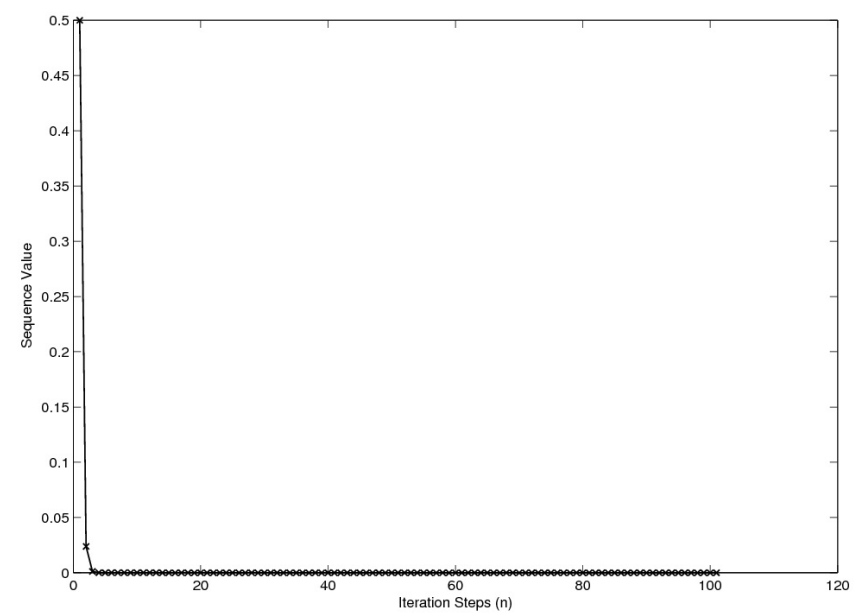

Figure 4. This figure shows the graph of the above table, we can see that $x_{n}$ converges to zero. 


\section{Acknowledgements}

We would like to thank the Faculty of Science, King Mongkut's University of Technology Thonburi (KMUTT) and the National Research Council of Thailand (NRCT-2555).

This research is finished at Department of Mathematics Education, Gyeongsang National University. We are very grateful to Professor Yeol Je Cho for his kindness and most helpful comments.

\section{References}

[1] H. Brezis , Operateurs maximaux monotones et semi-groups de contractions dans les espaces de Hilbert, NorthHolland Mathematics Studies, 1973. 2.4

[2] L.C. Ceng and J.C. Yao, A hybrid iterative scheme for mixed equilibrium problems and fixed point problems, Journal of Computational and Applied Mathematics, 214 (2008), 186-201. 1, 1

[3] P.L. Combettes and S.A. Hirstaoga, Equilibrium programming in Hilbert spaces, Journal of Nonlinear Convex Analalysis, 6 (2005), 117-136. 1

[4] P.L. Combettes and S.A. Hirstoaga, Equilibrium programming using proximal-like algorithms, Mathematics Programming, 78 (1996), 29-41. 1

[5] J.S. Jung, Iterative algorithms with some control conditions for quadratic optimizations, Panamer. Math. J., 16 (2006), 13-25. 2.5. 2.6

[6] J.S. Jung, A general iterative scheme for $k$ - strictly pseudo-contractive mappings and optimization problems, Applied Mathematics and Computation, 217 (2011), 5581-5588. 2.5. 2.6

[7] P. Kocourek, W. Takahashi, J.C. Yao, Fixed point theorems and weak convergence theorems for generalized hybrid mappings in Hilbert spaces, Taiwanese Journal of Mathematics, 14 (2010), 2497-2511. 1

[8] B. Lemaire, Which fixed point does the iteration method select? Recent advances in optimization (Trier, 1996), , Lecture Notes in Economics and Mathematical Systems, Springer, berlin, 452 (1997), 154-167. 2

[9] A. Moudafi, Viscosity approximation methods for fixed-point problems, Journal of Mathematical Analysis and Applications, 241 (2000), 46-55. 1

[10] M.A. Noor, Generalized set-valued variational inclusions and resolvent equation, Journal of Mathematical Analysis and Applications, 228 (1998), 206-220. 1.1

[11] J.W. Peng, Y. Wang, D.S. Shyu, J.C. Yao, Common solutions of an iterative scheme for variational inclusions, equilibrium problems and fixed point problems, Journal of Inequalities and Applications, vol. 2008, Article ID 720371, 15 pages. 1

[12] J.W. Peng, J.C. Yao, A new hybrid-extragradient method for generalized mixed equilibrium problems, fixed point problems and variational inequality problems, Taiwanese Journal of Mathematics, 12 (2008), 1401-1432. 1, 2.1.

[13] S.M. Robinson, Generalized equation and their solutions, part I: basic theory, Mathematical Programming Studies, 10 (1976), 128-141. 1.2

[14] R.T. Rockafellar, Monotone operators and the proximal point algorithm, SIAM Journal on Control and Optimization, 14 (1976), 877-898. 1.2

[15] T. Suzuki, Strong convergence of Krasnoselskii and Mann's type sequences for one parameter nonexpansive semigroups without Bochner integrals, Journal of Mathematical Analysis and Applications, 305 (2005), 227-239. 2.2. 2.3

[16] S. Takahashi, W. Takahashi, Strong convergence theorem for a generalized equilibrium problem and a nonexpansive mapping in a Hilbert space, Nonlinear Analysis: Theory, Methods and Applications, 69 (2008), 1025-1033. 1 , 1

[17] S. Takahashi, W. Takahashi, Viscosity approximation methods for equilibrium problems and fixed point problems in Hilbert spaces, Journal of Mathematical Analysis and Applications, 331 (2007), 506-515. 1

[18] H.K. Xu, Iterative algorithms for nonlinear operators, Journal of London Mathematical Society, 66 (2002), 240256.

[19] Y. Yao, Y.J. Cho, and Y.C. Liou, Iterative algorithms for variational inclusions, mixed equilibrium and fixed point problems with application to optimization problems, Central European Journal of Mathematics, 9 (2011) 640-656. (document) 1. 1

[20] Y. Yao, Y.C. Liou, J.C. Yao, Convergence theorem for equilibrium problems and fixed point problems of infinite family of nonexpansive mappings, Fixed Point Theory and Applications, vol. 2007, Article ID 64363, 12 pages. 1

[21] Y. Yao, Y.C. Liou, C. Lee, M.M. Wong, Convergence theorem for equilibrium problems and fixed point problems, Fixed Point Theory, 10 (2009), 347-363. 1

[22] S.S. Zhang, J.H.W. Lee, C.K. Chan, Algorithms of common solutions for quasi variational inclusion and fixed point problems, Applied Mathematics and Mechanics, 29 (2008) , 571-581, . 